\title{
REACTION OF SERA FROM INFERTILE FEMALE PATIENTS WITH FRACTIONATED PHYLOGENETICALLY CONSERVED OVARIAN ANTIGENS MEASURED BY ELISA: A PILOT STUDY
}

\section{Stefka M. Delimitreva, Ralitsa S. Zhivkova, Irina V. Chakarova, Valentina P. Hadzhinesheva, Vladislav V. Lazarov, Dimitrina K. Dimitrova- Dikanarova}

Department of Biology, Faculty of Medicine, Medical University of Sofia, Bulgaria

\author{
Corresponding Author: \\ Stefka M. Delimitreva \\ Department of Biology, \\ Faculty of Medicine, \\ Medical University of Sofia \\ 1, Sv. Georgi Sofiiski Str. \\ Sofia, 1431 \\ Bulgaria \\ e-mail:delimitreva@yahoo.com
}

Received: June 06, 2017

Revision received: July 07, 2017

Accepted: November 02, 2017

\section{Summary}

The reaction of anti-sperm antibody-positive sera from infertile women with fractionated mouse ovarian antigens was measured by enzyme-linked immunosorbent assay (ELISA). Antigens were obtained by extraction for nuclear matrix and intermediate filaments (NM-IF) producing three protein fractions - soluble, cytoskeletal and NM-IF. The results showed that sera from some infertile patients, but not control sera, react with either the soluble fraction or the NM-IF fraction. The reaction with soluble proteins was most likely directed against surface antigens, possibly aggravating the fertility problems, while the anti-NM-IF antibodies could indicate release of insoluble intracellular components by tissue damage of unknown origin.

Key words: nuclear matrix, intermediate filaments, antiovarian antibodies, immunological infertility, ELISA

\section{Introduction}

In about a quarter of patients seeking treatment for infertility, its cause remains unexplained. Among women with such unexplained infertility, more than $20 \%$ have enhanced humoral immune response. It includes both isoantibodies (anti-sperm) and autoantibodies (anti-ovarian, anti-phospholipid etc.), suggesting that these antibodies could be a causative factor [1]. While anti-sperm antibodies could jeopardize fertilization, anti-ovarian antibodies could act at an even earlier stage by interfering with oogenesis. It is therefore of interest which ovarian components are most immunogenic and become targets of autoantibodies. The first step to their identification is to fractionate ovarian tissue and to test how positive sera of female patients react with different fractions. Because of the technical and ethical difficulties in obtaining human ovarian tissue, ovaries from other mammals could be used. Previous studies on anti-sperm antibodies have shown that the relevant autoantigens are phylogenetically conserved across various mammalian orders [2], and a similar conservation could be expected for at least a proportion of the ovarian antigens.

A suitable method to fractionate a tissue based on the position and properties of its components is the extraction for nuclear matrix and intermediate filaments (NM-IF) was first described by Fey et al. [3]. Cellular proteins are separated into three fractions: 
soluble, cytoskeletal (including microtubule and microfilament components) and NM-IF. Initially developed for cultured epithelial cells, the method has been successfully applied on spermatozoa [4, 5] and then on oocytes and oocyte-cumulus complexes $[6,7]$. The aim of the present study was to find which (if any) fractions of mouse ovarian tissue are recognized by sera from women with unexplained infertility and enhanced humoral immune response. The reaction was measured by enzyme-linked immunosorbent assay (ELISA).

\section{Materials and Methods}

All work with patients and animals was carried out in accordance with Bulgarian and European Union legislation and was approved beforehand by the Ethical Committee of the Medical University of Sofia.

Mouse ovaries were obtained from 4-5-weekold BALB/c females. After mechanical homogenization, the tissue was subjected to NM-IF extraction as described by Markova [5]. The tissue was first treated with a detergent to extract soluble cytoplasmic proteins as well as membrane proteins. The supernatant was the soluble fraction. The pellet was extracted by high ionic strength of $250 \mathrm{mM}\left(\mathrm{NH}_{4}\right)_{2} \mathrm{SO}_{4}$ to dissolve microtubules and microfilaments. The protein content was further fractionated into supernatant (cytoskeletal fraction) and pellet (NM-IF fraction).

The study subjects were women from couples suffering of infertility as defined according to the World Health Organization criteria [8] for which no infertility factor was identified in the male partner. The women were tested for anti-sperm antibodies at the Laboratory of Reproductive Immunology, Department of Biology, Medical University of Sofia, with ELISA [9]. For the purpose of testing for enhanced humoral immune response, serum samples, positive for anti-sperm antibodies, were taken, a total of eight sera. The patients, aged 25-37, gave informed consent. Sera from six healthy age-matched female blood donors were used as controls.

ELISA was done as in [9] with small modifications. The three fractions of ovarian tissue, diluted with carbonate buffer $(\mathrm{pH} 9.6)$ to a final protein concentration of $0.2 \mathrm{mg} / \mathrm{ml}$, were used as an antigen. After loading the plates, they were treated with bovine serum albumin $(1 \%)$ as a blocking reagent. Then, the patient and control sera were diluted 1:10 and applied in triplicates. The secondary antibody was antihuman peroxidase conjugate (Sigma-Aldrich) diluted 1:1000. After the color reaction with o-phenylenediamine as substrate, absorbance was measured at $492 \mathrm{~nm}$. Wells without antigen and without primary antibody were left as negative controls. The arithmetic mean and the standard deviation were calculated for the control sera, and values exceeding the mean plus 2 standard deviations were considered positive.

\section{Results}

None of the six control sera from healthy donors produced a positive reaction. Of the eight patients' sera, four did not produce a positive reaction with any ovarian antigen fraction, similarly to the controls. The other four patients' sera reacted positively with one antigen fraction each. Two of them produced a positive result with the soluble fraction and two - with the NMIF fraction.

\section{Discussion}

The current study is the first to investigate the reaction of sera from infertile women with phylogenetically conserved ovarian antigens after NM-IF extraction. By using anti-sperm antibody positivity as an inclusion criterion, we intended to select sera with elevated humoral immune response. This could be expected to result in non-specific enhancement of the reaction with a broad range of antigens. However, half of the tested patients' sera were not found to contain anti-ovarian antibodies. The other half (four sera) reacted with ovarian antigens, but none of them produced a positive result with more than one antigen fraction. Hence, the tested sera showed antigen specificity rather than a broad range of reaction. It is noteworthy that no serum bound the cytoskeletal fraction, which could be interpreted as low immunogenicity of microtubule and microfilament components.

The positive sera reacted with soluble or NM-IF ovarian antigens with equal probability. Considering the possible contribution of serum antibodies to infertility, reaction with the soluble fraction was most important, because it included 
the surface proteins. Their binding by antibody in vivo could result in inhibitory or cytotoxic action and so affect the ovarian function. At the same time, reaction with the NM-IF antigens - the most stable and extraction-resistant fraction, should not be dismissed as irrelevant. When reacting with sperm cells, the positive sera most likely recognized the NM-IF fraction as well, which includes proteins present also in the ovary such as vimentin [10] and cytokeratins [11] as well as highly resistant cell type-specific components [12]. Possible factors for the immunogenicity of NM-IF proteins are their fibrous structure and low solubility resulting in difficult clearance. Antibodies to such intracellular components are not expected to inflict any damage on the target cells, but could indicate cell destruction caused by other unidentified processes that release NMIF proteins and trigger iso- and autoimmune humoral immune response. Because of the small number of the sera, the current study is of a preliminary type and further investigations are needed.

\section{Conclusions}

Our study showed that some anti-sperm antibody-positive sera from infertile women, but not control sera, react with fractionated phylogenetically conserved ovarian antigens after NM-IF extraction. Immunogenic properties were observed for the soluble and the NM-IF fractions. The first category of antibodies were most likely against surface antigens, possibly aggravating the fertility problems, while the anti-NM-IF antibodies could indicate release of insoluble intracellular components by tissue damage of unknown origin.

\section{Acknowledgements}

This work was supported by the Medical University of Sofia grant No. 36/2012.

\section{References}

1. Buteva-Hristova I, Lazarov V, Lozanov V, Gateva A, Bechev B, Kavaldzieva K, et al. Serum anti-alpha-crystallin antibodies in women with endocrine disorders. Biotechnol Biotechnol Equip. 2017; 31(3):574-80. doi: 10.1080/13102818.2017.1308232.
2. Fichorova R, Mavrov C, Mladenov I, Dimitrova E, Nakov L. A novel approach to the generation of antibodies against phylogenetically preserved sperm antigens. Theriogenology. 1996;45(5):957-75.

3. Fey EG, Wan KM, Penman S. Epithelial cytoskeletal framework and nuclear matrixintermediate filament scaffold: threedimensional organization and protein composition. J Cell Biol. 1984;98(6):1973-84.

4. Markova MD. Electron microscopic observations of mouse sperm whole mounts after extraction for nuclear matrix and intermediate filaments. Arch Androl. 2001;47(1):37-45.

5. MarkovaMD.Electronmicroscopicobservations of human sperm whole-mounts after extraction for nuclear matrix and intermediate filaments (NM-IF). Int J Androl. 2004;27(5):291-5.

6. Kadam KM, D‘Souza SJ, Bandivdekar AH, Natraj U. Identification and characterization of oviductal glycoprotein-binding protein partner on gametes: epitopic similarity to nonmuscle myosin IIA, MYH 9. Mol Hum Reprod. 2006;12(4):275-82.

7. Nikolova V, Delimitreva $\mathrm{S}$, Zhivkova $\mathrm{R}$, Chakarova I, Dimitrova D, Markova M. Cytoskeletal changes during mouse oocyte maturation revealed by a variation of nuclear matrix and intermediate filaments (NM-IF) extraction. CR Acad. Bulg. Sci. 2011;64(11):1571-6.

8. Zegers-Hochschild F, Adamson GD, de Mouzon $\mathrm{J}$, Ishihara O, Mansour R, Nygren $\mathrm{K}$, et al. The International Committee for Monitoring Assisted Reproductive Technology (ICMART) and the World Health Organization (WHO) revised glossary on ART terminology, 2009. Hum Reprod. 2009;24(11):2683-7.

9. Dimitrova D, Kalaydjiev S, Hristov L, Nikolov K, Boyadjiev T, Nakov L. Antichlamydial and antisperm antibodies in patients with chlamydial infections. Am J Reprod Immunol. 2004;52(5):330-6.

10. Markova M, Nikolova V, Marinova T. Presence and asymmetric distribution of cytoskeletal protein vimentin in rat sperm head. Andrologija (Sofia). 2009;18(1)3-5.

11. Markova M, Nikolova V, Marinova $\mathrm{T}$. Immunocytochemical localization of cytokeratins in human sperm head. Andrologija (Sofia). 2010;19(2)14-6.

12. Markova MD, Nikolova VP, Marinova TT. Observations of interface between tail microtubules and outer dense fibers in human necrozoospermic spermatozoa. Journal of Biomedical and Clinical Research. 2011;4(2):82-5. 